\title{
Dynamic Data update for Intelligent Speed Adaptation (ISA) System
}

\author{
B.Karthikeyan \\ Embedded System Division \\ School of Electronics Engineering \\ VIT University, Vellore
}

\author{
M. Tamileniyan \\ Post Graduate Engineering Trainee \\ Automotivelnfotronics
}

\begin{abstract}
The Intelligent Speed Adaptation in vehicles using GPS technology is a method of speed adaptation in vehicles based on its position on the highway. The position can be recognized using the GPS receiver. The main objective is to make the system simple. The Toll gates on the highway are equipped with a transmitter that transmits a very small data to the receiver system in the vehicle. The received data carries a table of information with the latitude-longitude and its speed limit. As soon as the system receives the signal, the system starts operating; the system checks the latitude longitude data with the current position. When the system reaches the position, the controller informs the driver about the speed limit and also limits the speed of vehicle to the speed limit levels. Simultaneously the second data is taken from the received data and checks for next position and so on, till the last data. After the given data is executed, the next toll gate will be approaching for the speed limit patterns till the next tollgate. This system reduces usage of memory that is used for storing large map information, also reduces the cost of implementation in vehicles.
\end{abstract}

Key words: ZigBee, Dynamic Data, Speed Adaptation

\section{INTRODUCTION}

Intelligent Speed Adaptation (ISA), also known as Intelligent Speed Assistance, is any system that constantly monitors vehicle speed and the local speed limit on a road and implements an action when the vehicle is detected to be exceeding the speed limit. This can be done through an advisory system, where the driver is warned, or through an intervention system where the driving systems of the vehicle are controlled automatically to reduce the vehicle's speed.

Intelligent speed adaptation uses information about the road on which the vehicle travels to make decisions about what the safe speed should be. This information can be obtained through use of a digital maps incorporating roadway coordinates as well as data on the speed zoning for that roadway at that location, through general speed zoning information for a defined geographical area (e.g., an urban area which has a single defined speed limit), or through feature recognition technology that detects and interprets speed limit signage. The purpose of ISA is to assist the driver in keeping to the lawful speed limit at all times, particularly as they pass through different speed 'zones'. This is particularly useful when drivers are in unfamiliar areas or when they pass through areas where variable speed limits are used.

Most drivers do not appreciate the extra risks involved in traveling just a few $\mathrm{km} / \mathrm{h}$ over the speed limit. Most think that the risk of a casualty crash is doubled if traveling at least 25 $\mathrm{km} / \mathrm{h}$ over the speed limit. Research has found that that, in urban areas, the risk of a casualty crash is doubled for each $5 \mathrm{~km} / \mathrm{h}$ over the limit. So traveling at $70 \mathrm{~km} / \mathrm{h}$ in a $60 \mathrm{~km} / \mathrm{h}$ zone quadruples the risk of a crash in which someone is hospitalized. As a result, it is estimated that about $10 \%$ of casualties could be prevented if the large group of motorists who routinely travel at up to $10 \mathrm{~km} / \mathrm{h}$ over the limit were encouraged to obey the speed limits. About $20 \%$ of casualties could be prevented if all vehicles complied with the speed limits. Savings in fatal crashes would be larger.

There are many types of ISA systems of which GPS based ISA is the most recent and effective system. A GPS receiver is used with the ISA system to detect the vehicle's current location on the earth as coordinates, and this coordinates are use to find its location by a preloaded map which contains the information about the roads and its various highway signals and speed limits. Event though this system is recent and effective, there are few limitations in this system and that is why this project is done to reduce to cost of the existing system, to improve the reliability and to make the system have updated information, dynamically.

\subsection{Existing Speed Adaptation systems}

There are number of Speed Adaptation systems proposed, at different times, but they are not effective and resulted in failures. Some important Speed Adaptation Systems are

\subsubsection{RFid - Radio beacons}

Roadside radio beacons, work by transmitting data to a receiver in the car. The beacons constantly transmit data, which the carmounted receiver picks up as it passes each beacon. This data could include local speed limits, school zones, variable speed limits, or traffic warnings. If sufficient numbers of beacons were used and were placed at regular intervals, they could calculate vehicle speed based on how many beacons the vehicle passed per second. Beacons could be placed in/on speed signs, telegraph poles, other roadside fixtures, or in the road itself. Mobile beacons could be deployed in order to override fixed beacons for use around accident scenes, during poor weather, or during special events. Beacons could be linked to a main computer so that quick changes could be made.

This system can be used only for slow vehicles. Practically it cannot be used in the highway for vehicles with high speed. When vehicle moves with high speed on the road, the data will not be picked up by the car unit, and hence this system was not effective

\subsubsection{Optical recognition systems}

This system uses a camera mounted on the car to continuously capture the images on the road. The image is processed to find if 
there is any sign board present on the image, this is done by digital image processing to detect the shape of the signboard. Once the sign board is found, the other algorithm to find the pattern in the image in the signboard is used to extract the symbol and compare to recognize the sign board. According to the symbol recognized, information is given to the driver either by a LCD display on the dashboard or by buzzer alert or even control the vehicle automatically to strict to highway rules. It should be noted that all these process must be carried out within a fraction of second. It was suggested to keep the visual sign boards more away from the point. For eg. Now if the signboard is placed $50 \mathrm{~m}$ away from the speed breaker, it was suggested to keep the signboard a $100 \mathrm{~m}$ away so that the system has enough time to process and driver has enough time to control the vehicle.

- This system uses complex algorithm

- Uses much resources

- Some critical cases when the sign board is covered by tree branches, the system does not detect the sign board.

\subsubsection{Dead reckoning}

Dead reckoning (DR) uses a mechanical system linked to the vehicle's driving assembly in order to predict the path taken by the vehicle. By measuring the rotation of the road wheels over time, a fairly precise estimation of the vehicle's speed and distance traveled can be made. Dead reckoning requires the vehicle to begin at a known, fixed point. Then, by combining speed and distance data with factors such as the angle of the steering wheel and feedback from specialized sensors (e.g., accelerometers, flux gate compass, gyroscope) it can plot the path taken by the vehicle. By overlaying this path onto a digital map, the DR system knows approximately where the vehicle is, what the local speed limit is, and the speed at which the vehicle is traveling. The system can then use information provided by the digital map to warn of upcoming hazards or points of interest and to provide warnings if the speed limit is exceeded.

This system is much complicated with multiple sensors and possibility of deviation from actual position is high

\subsubsection{GPS based systems}

GPS is based on a network of satellites that constantly transmit radio signals. GPS receivers pick up these transmissions and compare the signals from several satellites in order to pinpoint the receiver's location to within a few meters. This is done by comparing the time at which the signal was sent from the satellite to when it was picked up by the receiver. The GPS receiver module tracks its position and using the pre loaded map with speed zone database, the speed adaptation is done.

- Requires a large memory to store the complete map and speed zone databases.

- Emergency sign boards like "MEN AT WORK”, "TAKE DIVERSION" etc. are not updated to the system dynamically.

\subsection{Need for a new system}

- To over come the limitations of the other Speed adaptation systems.

- To reduce the memory requirement of conventional GPS speed adaptation techniques.

- To reduce the system cost
- To enhance the system performance to provide dynamic update of information.

\section{SYSTEM DESCRIPTION}

\subsection{System Overview}

The Intelligent Speed Adaptation system with Dynamic data update contains the basic control system for conventional GPS based ISA system with a GPS receiver to locate their vehicle's geographic location and a wireless transceiver to receive the data which is a table of coordinates and speed limit.

\subsubsection{Overall System Operation}

The overall system contains the essential components of the ISA system with a wireless Transceiver. The tollgates in the highway have a Transceiver that is capable of communicating with the vehicle's transceiver. The tollgate sends a data continuously around its coverage range. When the Vehicle which is equipped with the proposed system arrives near the tollgate, the data broadcasted by the tollgate is received in the vehicle module. This data is stored into the memory of the ISA system. The data sent is a table of information of coordinates and speed limit. That is, it contains the latitude and longitude value of a location and its corresponding speed limit or highway traffic symbols.

As soon as the data is stored in the memory, The GPS receiver tracks it location and compares its coordinates with the table of coordinates received, as soon as the first value of coordinates and the current coordinates from GPS matches, the corresponding speed limit listed in the table is chosen as the speed limit from that point. The ISA system then alerts the driver by sound alert, displaying the speed limit and indicates the speed limit of the location.

\subsection{Algorithm}

Step 1: The Vehicle's wireless device is $\mathrm{ON}$ as soon as the vehicle is started, and waits for the data to be received.

Step 2: When Vehicle approaches the tollgate in the highway, and when it comes in to the tollgate's wireless coverage area, the communication link is established

Step 3: Based on the communication link and direction, the data is sent to the vehicle's wireless node. The data is stored into the memory stack.

Step 4: $\quad$ The stored data is the table of information based on geographic coordinates of the vehicle (longitude \& latitude) and corresponding speed limits and highway signs are arranged in proper sequence.

Step 5: Stack data is compared with the received data, when received data is equal to the stack data, the corresponding is used.

Step 6: The sign is used as the current sign for the position, and corresponding action is done, either to display or alert or control the vehicle.

Step 7: Now, the second row data in the table is selected and compared with current location again.

\subsection{Dynamic Data Update System}

The Dynamic Data Update System for the ISA system includes a RF Transceivers in the vehicle and RF transceivers in the tollgates of the highway. The RF transceivers on the tollgate 
should be able to communicate to any vehicle with the transceiver. When a car approaches near to the tollgate, as soon as the both transceiver comes into its mutual coverage zone, the data transaction is initiated and thus, the data from the tollgate is sent to the vehicle, and that data is further saved into the memory of the ISA system.

The main objective of the Dynamic Data Updates system used is to updated information about the road situation to the vehicle dynamically. Usually in GPS based ISA systems, the road map and the speed limit data are very old, and many times, new road condition detail are not available in the vehicle's ISA database. Hence updating the information from Tollgate will provide latest detail, and provide emergency indications like "Take diversion", "Men at work". "Accident zone, drive slow", etc.

\section{THEORY}

ZigBee is chosen as the best wireless technology to transmit the data from the tollgate to the vehicle, which is the most important part of this project. One ZigBee module is fixed in the vehicle with the ISA system, and one or more ZigBee transceivers are placed in the tollgate to send data to multiple vehicles in either direction.

\subsection{Introduction to ZigBee}

- ZigBee is a standard that defines a set of communication protocols for low-data-rate, short-range wireless networking.

- ZigBee-based wireless devices operate in $868 \mathrm{MHz}, 915 \mathrm{MHz}$, and $2.4 \mathrm{GHz}$ frequency bands. The maximum data rate is 250 $\mathrm{K}$ bits per second.

- ZigBee is targeted mainly for battery-powered applications where low data rate, low cost, and long battery life are main requirements

\section{HARDWARE TEST SETUP}

The Hardware Test setup contains the basic configuration for communication between two ZigBee nodes in point to point to point network structure. Two ZigBee modules are used here, these modules are connected to two different computers through RS232 port. Considering the voltage lever variations between the computer's RS232 port and the ZigBee's CMOS level, a voltage level shifter is used. The MAX232 chip is used as a voltage level shifter or interface between the RS232 level and ZigBee's CMOS level.

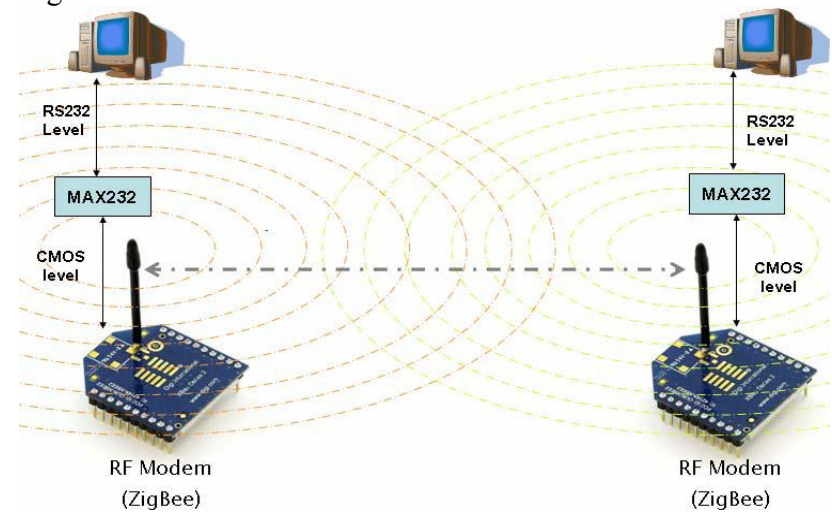

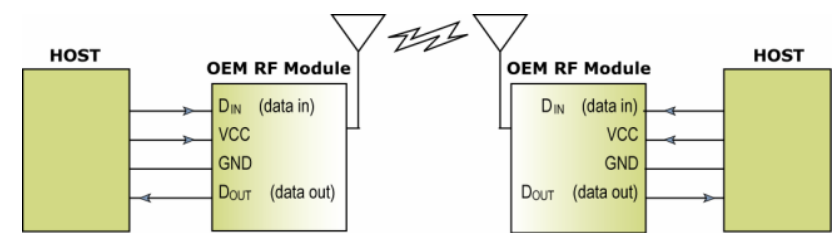

Fig.4. 10 Hardware Test setup

\section{TEST RESULTS}

The analysis was done in different environments and different files and configurations were used to test the Zigbee for application in Dynamic Data Update system. The detailed results for different environments are as follows.

\subsection{Results - Indoor Environment}

Analysis was done in Indoor environment for data transfer in indoor. Image files are used as the data for transmission. A set of files of different sizes and file formats were used to find the transmission time and number of packet losses.

\subsubsection{Different serial Baudrates}

Same set of files were used for different serial interface baud rates. The ZigBee module is attached to the host computer. The communication port is opened for the communication from ZigBee to computer using communication terminal in the computer. Necessary serial settings are configured and the serial baudrate setting of the ZigBee device is changed by the following procedure.

1. The communication terminal is opened.

2. Enter the AT command mode by pressing the escape characters (" +++ " for XBee)

3. Change the baudrate setting by typing the command ATBD <baudrate number>

4. Write the changes done by sending the command ATWR

5. Exit from AT command mode by sending ATCN

After baudrate change, the usual procedure of sending a file is done for analysis. Fig's 5.1, 5.2, 5.3, 5.4 and table 5.0 shows different baudrate analysis.

Table5.0 Analysis in different serial baudrates

\begin{tabular}{|l|l|l|l|l|}
\hline $\begin{array}{l}\text { Baudrate/file } \\
\text { size }\end{array}$ & $19 k b$ & $31 k b$ & $41 k b$ & $72 k b$ \\
\hline $9600 b p s$ & 28 & 67 & 77 & 134 \\
\hline $57600 b p s$ & 3 & 7 & 9 & 17 \\
\hline $115200 b p s$ & 2 & 3 & 4 & 7 \\
\hline
\end{tabular}


Fig. 5.1 At 9600 bps serial baudrate

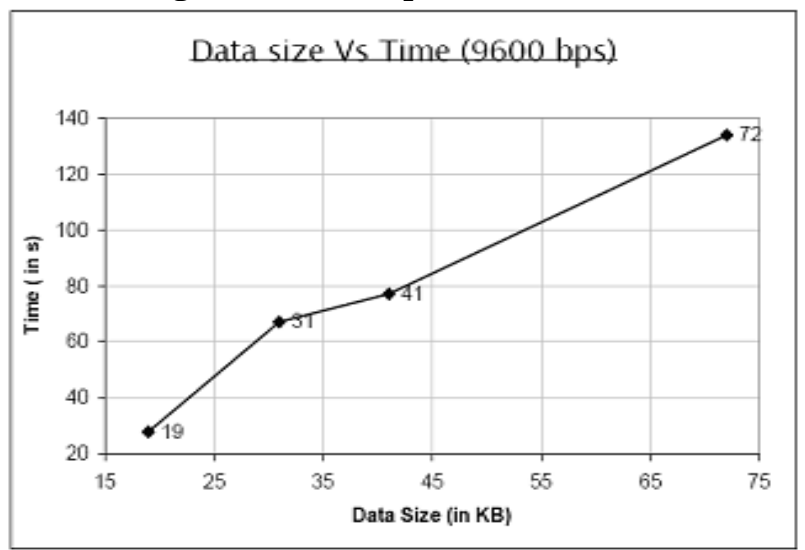

Fig. 5.2 At 57.6 Kbps serial baudrate

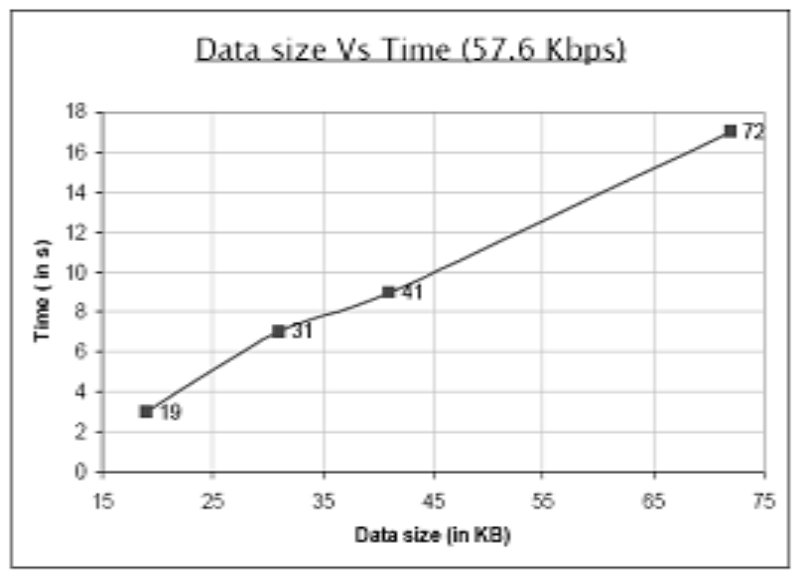

Fig. 5.3 At 115.2 Kbps serial baudrate

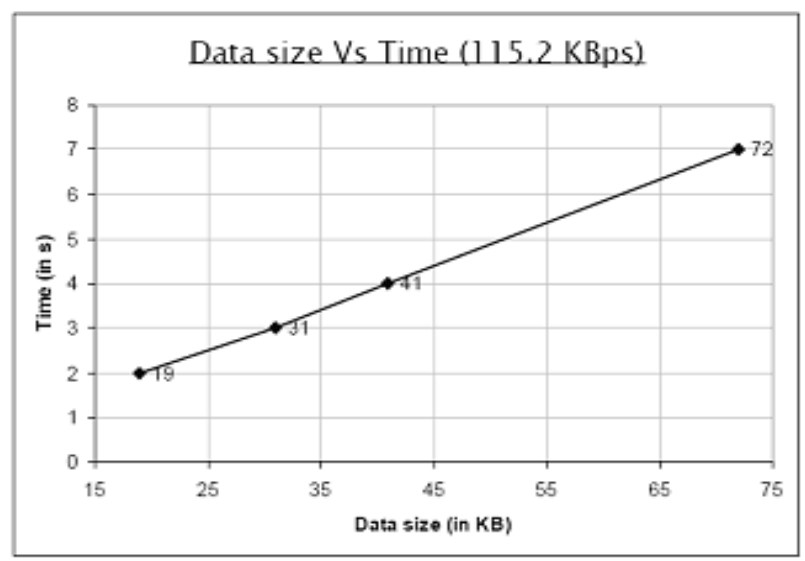

Fig. 5.4 Different file size

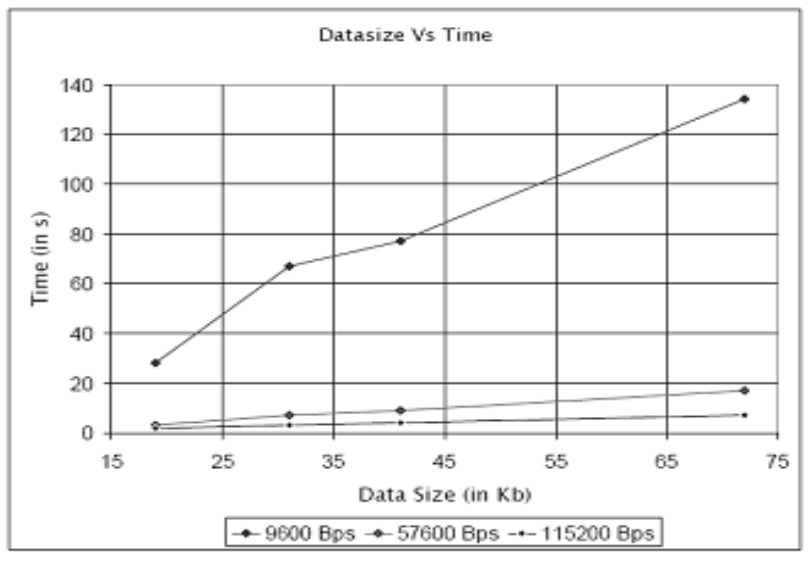

\subsubsection{Number of Packet loss}

When data transmission is done, it is noted that packet losses occur, The data when it is sent, if the packet does not reach the receiver perfectly, and if there is any loss in the bits, then retransmission of that packet takes place again. Hence this will increase the data transmission time. The number of retries made in the data transaction is proportional to the packet losses occurred.

Fig 5.5 Indoor environment

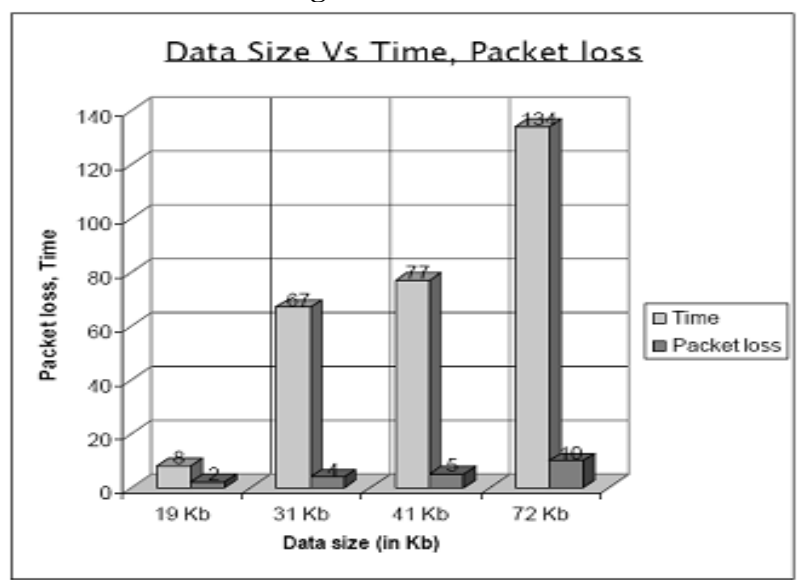

The fig 5.5 is the representation of data size Vs packet loss for a typical data transmission in indoor environment. It is observed that the packet losses increase as the file increases. Hence these packet losses make retransmission of the earlier packet and thus reducing the data transfer speed.

\subsection{Outdoor Environment}

The outdoor test was done using laptops, two interface boards with ZigBee modules are connected to laptops.

- A straight road is chosen

- Road length is measured; reference marks are made on the road for each test distance.

- Data transmission was analyzed by sending same set of data at different positions.

\subsubsection{Different distances}

Signal strength is analyzed by using the Received signal strength indicator (RSSI) option in the XBee. The XBee returns the 
signal strength in hex value of $\mathrm{dbm}$. This is converted to the signal level in dbm.

When signal strength is measured in fixed locations, it is observed that the signal strength is not exactly same at the particular position for every trial.

\subsubsection{Packetloss in outdoor}

In outdoor setup, the data transmission time is unpredictable. For every trial, the data transmission time taken and the number of retransmissions are different. But the speed of data transmission is more in outdoor, comparing to the indoor setup.

Fig 5.6 Outdoor analysis in different distances, different trials
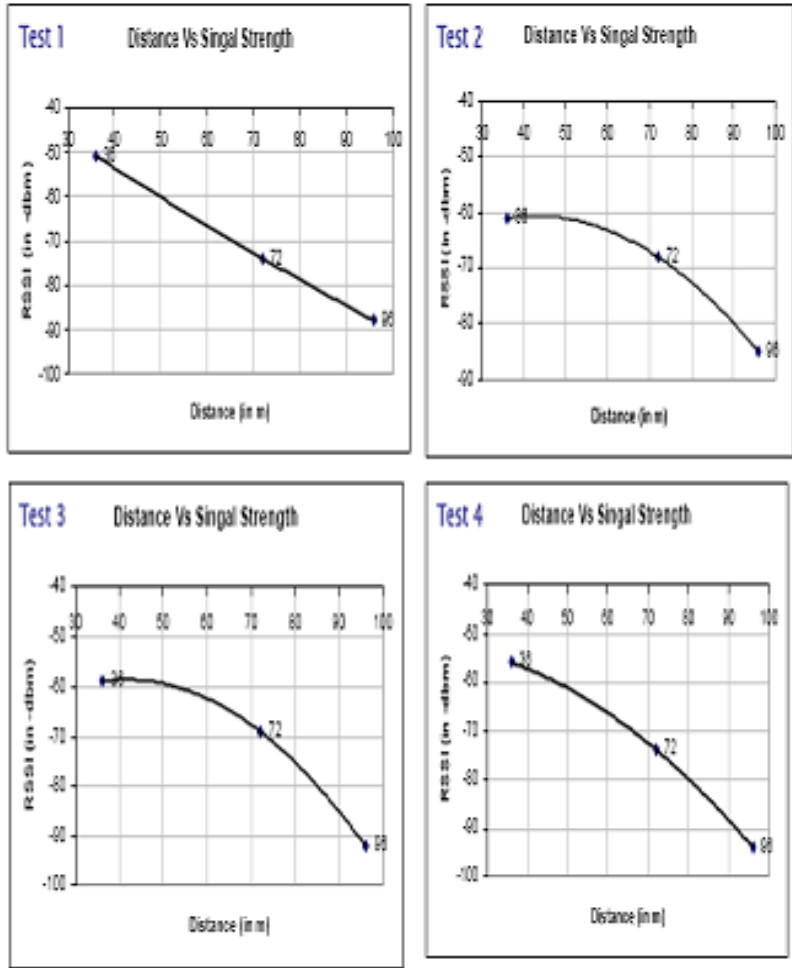

Fig 5.7 Outdoor analysis with different distances and different trials

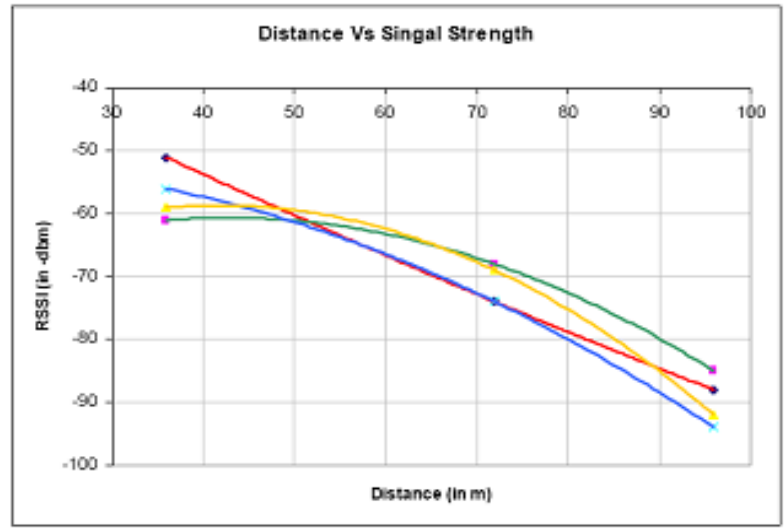

Fig 5.8 Data transfer at 31Kbat 9600bps for outdoor

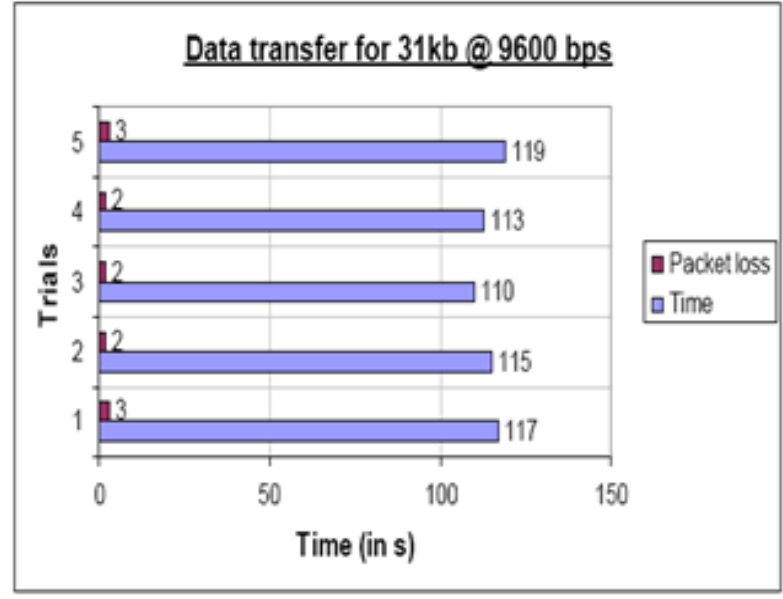

\subsection{Dynamic Environment}

In dynamic Environment, ZigBee modules are interfaced with laptop for convenience; the same setup used for the outdoor is used. But here, one module is kept fixed a position. Other module is placed in a vehicle. Both the modules are powered on, and when one module approaches the other, when it comes into the coverage zone, the data transmission is started while moving, and data will be sent to the moving device.

Observations: The coverage area around a ZigBee node was found to be around $70 \mathrm{~m}$. But when one module attached to a vehicle moves approaching the other fixed module. The communication link is established only when it gets very close to the fixed module. Hence there exists a reconnection problem, when a device out of connection newly enters the coverage zone. Hence for reconnection, it requires the signal strength to be at maximum. So, only when

the device is around $10 \mathrm{~m}$ close to each other, the communication link is established. Once the connection is done, and data transfer is started, the connection exists for next $40 \mathrm{~m}$, by the time, the data is transmitted.

\subsection{Image data verification using MATLAB}

An image file is sent from one end to the other, since this is sent through air, there are chances of data loss or bit corruption. When there is a block of bits missed or corrupted, the image will have the indication directly, but if there is a one bit variations, the image will look same but there will be a slight color variation in that particular pixel. So the sent image and received image are taken, they are compared in MATLAB by separating the RGB matrix of each and comparing each and every element of the matrix.

Observations: On comparing, it is observed tat all images sent are received perfectly and relevance of sent and received image is $100 \%$ and $0 \%$ difference.

\section{CONCLUSION}

An analysis on IEEE for ZigBee was done for application in Dynamic Data Update system. Experiments were done for data transmission in different environments like indoor, outdoor, and 
dynamic. In indoor setup, the data transmission time and packet loss was predictable and same for number of trials. ZigBee is tested for coexistence with other wireless technologies wifi, Bluetooth that operates in the same frequency band. The data transmission had no effect on the wifi or Bluetooth environment. In outdoor setup, the data transmission and packet loss was unpredictable. The transmission time was not same for every trial. But data transmission speed was more in outdoor environment, sometimes.

In dynamic setup, Data transmission takes place only when the two nodes are very close. Normally, the coverage area around ZigBee node was approximately $80 \mathrm{~m}$. When one node is fixed and other node approaches the fixed node, data transmission starts only when the distance between both the nodes comes closer to $10 \mathrm{~m}$. This shows that, when node enters the ZigBee coverage zone, to establish the communication link, it requires maximum signal strength. Once data transmission is started when distance is $10 \mathrm{~m}$, the communication link exists between for next $30 \mathrm{~m}$ away from the fixed node, in which the data can be transmitted. Hence, in Dynamic Data Update System, it can be suggested to fix the ZigBee node, $10 \mathrm{~m}$ before the tollgate to use the available short time effectively to transmit the data.

\subsection{Future works}

To avoid communication problem in dynamic environment, a ZigBee module can be configured in Router role. And thus this router can be placed a few meters before the main device to make more coverage area and more time for data transfer. Many ZigBee modules can be configured as routers and placed around the tollgate in different location, by which the location of the ZigBee module can be traced by comparing RSSI of every node. This will be useful for Dynamic Data Update system to find the direction and position of vehicle.

\section{REFERENCES}

[1]. N. Agerholm,R, Waagepetersen,N. Tradisauskas,and H. Lahrmann 2008 - IEEE Intelligent Vehicles Symposium "Intelligent Speed Adaptation in Company Vehicles".
[2]. Riaz Sayed, Pierre Delaigue, Jeremy Blum, Ali Mortazavi and Azim Eskandarian 2007-SAE TECHNICAL PAPER SERIES "Development of an Intelligent Multimode Speed Adaptation System"

[3]. Georgiy Pekhteryev, Zafer Sahinoglu, Philip Orlik, and Ghulam Bhatti 2005 - MITSUBISHI ELECTRIC RESEARCH LABORATORIES "Image Transmission over IEEE 802.15.4 and ZigBee Networks"

[4]. Dr.S.S.Riaz Ahamed-2008 -Journal of Theoretical and Applied Information Technology "The role of ZigBee technology in future data Communication system"

[5]. Marko Hannikainen, Timo D. Hamalainen, Markku Niemi, Jukka Saarinen Computer communications "Trends in personal wireless data communications"

[6]. Jesus Tellez Isaac, Jose Sierra Camara , Sherali Zeadally, Joaquin Torres Marquez- March 2008 Computer communications. "A secure vehicle-to-roadside communication payment protocol in vehicular ad hoc networks"

[7]. European Road Safety Observatory "Intelligent Speed Adaptation http://www.erso.eu/knowledge/content/04_esav

[8]. University of Leeds and MIRA Ltd. - January 2006 "Intelligent Speed Adaptation Literature Review and scoping study"-http://www.tfl.gov.uk/assets

[9]. H. Labiod, h. Afifi, c. De santis - Springer publications "Book on Wi-Fi, Bluetooth,,ZigBee and Wimax"

[10]. Shahin Farahani - Elsevier publications "ZigBee Wireless Networks and Transceivers"

[11]. ZigBee product descriptions "XBee product manufacturerDigi International". http://www.digi.com/technology/rfarticles/wireless-zigbee.jsp 